\title{
POSITIVE SOLUTIONS OF POSITIVE LINEAR EQUATIONS
}

\author{
PAUL NELSON, JR.
}

\begin{abstract}
Let $B$ be a real vector lattice and a Banach space under a semimonotonic norm. Suppose $T$ is a linear operator on $B$ which is positive and eventually compact, $y$ is a positive vector, and $\lambda$ is a positive real. It is shown that $(\lambda I-T)^{-1} y$ is positive if, and only if, $y$ is annihilated by the absolute value of any generalized eigenvector of $T^{*}$ associated with a strictly positive eigenvalue not less than $\lambda$. A strictly positive eigenvalue is a positive eigenvalue having an associated positive eigenvector. For the case of $B=L^{p}$ this yields the result that $(\lambda I-T)^{-1} y \geqq 0$ if, and only if, $y$ is almost everywhere zero on a certain set which depends on $\lambda$ but is otherwise fixed.
\end{abstract}

In some fields of applied mathematics (e.g., radiative transfer, neutron transport) there occur conditional equations of the form

$$
\lambda x=\boldsymbol{T} x+y
$$

in which the parameter $\lambda$, the known element $y$, and the linear operator $\boldsymbol{T}$ are all positive, in the respective appropriate senses, and one wishes, for physical reasons, to conclude existence of a positive solution, $x$. The theorem given below has an obvious application to such problems. Its statement and proof are the primary purpose of this note.

Before stating the result, we describe the setting within which (1) is considered. The terminology and notation used is that of Day [1]. Let $B$ be a real Banach space, and denote by $K$ a closed cone in $B$ such that $B$ is a vector lattice under the partial order induced by $K$. We further suppose that the norm on $B$ has, relative to the order induced by $K$, the property termed semimonotonic by Krasnosel'skiı [2]; that is, there exists a positive real constant $M$ such that the situation $0 \leqq x \leqq y$ implies $\|x\| \leqq M\|y\|$. This property is the only connection which we require between the order and the norm. For $z \in B$ we denote $z^{+}+z^{-}=(z \vee 0)-(z \wedge 0)$ by $|z|$, with a similar notation for the conjugate space $B^{*}$. For $z^{*} \in B^{*}$ and $z \in B$, we will generally denote $z^{*}(z)$ by $\left(z, z^{*}\right)$. The linear operator $T$ is defined on $B$, is eventually compact ( $T^{n}$ is compact for some positive integer $n$ ),

Received by the editors March 16, 1971.

AMS 1970 subject classifications. Primary 45A05, 47B55; Secondary 46E30, 82A70, 82A75, 85A25.

Key words and phrases. Ordered Banach space, positive operator, positive solution, linear operator, neutron transport, radiative transfer, positive kernel. 
and is positive in the sense that $z \geqq 0$ implies $T z \geqq 0$. Let $\lambda_{1}>\lambda_{2}>$ $\cdots>0$ denote the set, either finite or countably infinite, of positive eigenvalues of $\boldsymbol{T}$ which have an associated positive eigenvector. The conjugate of $\boldsymbol{T}$ is denoted $\boldsymbol{T}^{*}$. Then the main result is as follows.

THEOREM. If $\lambda \geqq 0$ and $y \geqq 0$, then (1) has a solution $x \geqq 0$ if, and only if, $(y,|h|)=0$ for every $h \in B^{*}$ such that $\left(\lambda_{i}-T^{*}\right)^{p} h=0$ for some $\lambda_{i} \geqq \lambda$ and some positive integer $p$.

The remainder of this note is largely an outline of a proof of this theorem. If the $\left\{\lambda_{i}\right\}$ comprised all of the positive eigenvalues of $\boldsymbol{T}$, and if the condition $(y,|h|)=0$ were replaced by $(y, h)=0$, then the theorem would be an easy consequence of well-known results. However, it is precisely these extensions which yield the following interesting consequence of the theorem.

Corollary. Suppose $B=L^{p}(\mu), 1 \leqq p<\infty$, where the measure space underlying the measure $\mu$ is totally $\sigma$-finite if $p=1$. Then to each $\lambda_{i}$ there is a measurable set $S_{i}$, unique to within a null set, such that (1) has a nonnegative solution $x$ if, and only if, the representatives of $y$ are almost everywhere zero on $S_{i}$ for every $i$ such that $\lambda_{i} \geqq \lambda$.

The terminology of the corollary is that of Halmos [3]. The restrictions serve to give an adequate representation theory for the conjugate space of $B$ [4]. It suffices to take for $S_{i}$ the union of the sets of support of representatives of a maximal linearly independent set of equivalence classes $z$ satisfying $\left(\lambda_{i}-T^{*}\right)^{p} z=0$ for some positive integer $p$. A similar corollary holds for other spaces whose conjugates have a known representation.

In the case of neutron transport theory the sets $S_{i}$ can be interpreted as the subset of the system phase space such that source neutrons originating therein excite the critical state associated with $\lambda_{i}$. In this context the result is, roughly, of interest only for systems with loosely coupled parts, as otherwise the collection $\left\{\lambda_{i}\right\}$ can be shown to be a singleton set by, say, an adaptation of the treatment of Krein and Rutman [5] (see [6] for an example of a result of this type). Sufficient conditions for the integral operator governing neutron transport in a slab to be eventually compact in a certain $L^{1}$ space are given in [7].

The following simple lemma is the key to our proof of the above theorem.

Lemma. Let $\boldsymbol{Q}$ be a positive linear operator defined on $B$ and given by the formula

$$
\boldsymbol{Q} z=\sum_{i=1}^{n}\left(z, y_{i}^{*}\right) y_{i}
$$


where the $\left\{y_{i}\right\}$ are linearly independent. Suppose $z \geqq 0$. Then $Q_{z}=0$ if, and only if, $\left(z,\left|y_{i}^{*}\right|\right)=0$ for $i=1, \cdots, n$.

Proof. The direct assertion is obvious. For the converse, suppose, contrariwise, that there is some $z \geqq 0$ such that $Q z=0$ but $\left(z,\left|y_{i}^{*}\right|\right)>0$ for some $i$. Then we have $\left(z, y_{i}^{*+}\right)>0$. But [1, p. 98],

$$
\left(z, y_{i}^{*+}\right)=\sup \left\{\left(u, y_{i}^{*}\right) \mid 0 \leqq u \leqq z\right\},
$$

whence there is some $u$ such that $0 \leqq u \leqq z$ and $\left(u, y_{i}^{*}\right) \geqq\left(z, y_{i}^{*+}\right) / 2>0$. Thus $\boldsymbol{Q} u \neq 0$, and therefore $\boldsymbol{Q} u<0$ since $u \leqq z$ implies $\boldsymbol{Q} u \leqq \boldsymbol{Q} z$. But $\boldsymbol{Q} u \geqq 0$, since $u \geqq 0$ and $Q$ is positive. Thus we have a contradiction, and the lemma is proved.

We now turn to the proof of the theorem. First of all note that existence of a nonnegative solution, $x$, of (1) is equivalent to convergence of the Neumann series

$$
\sum_{n=0}^{\infty} T^{n} y / \lambda^{n+1} .
$$

If (2) converges then its sum is obviously such a solution. Conversely, any such solution must majorize the sequence of partial sums of (2). The sequence of partial sums is then norm bounded, by semimonotonicity, and therefore contains a convergent subsequence, by eventual compactness. But semimonotonicity implies that a monotone sequence which contains a convergent subsequence must be convergent itself.

We now replace $T$ and $B$ by their standard complexification, but without changing the notation. Now, for arbitrary fixed $y \in K$, there is some real number $a$ (=reciprocal radius of convergence) such that (2) converges if $|\lambda|>a$ and diverges if $|\lambda|<a$. Furthermore, by standard arguments of the type used in connection with the abstract Pringsheim's theorem [8], [9], $a$ is itself a singular point of the analytic function of $\lambda$ defined by (2). But this analytic function is $R_{\lambda} y, R_{\lambda}=$ resolvent of $T$. Thus $a$ is a spectral point of $T$, and hence an eigenvalue of $T$.

We now know that the reciprocal radius of convergence of (2) is some positive eigenvalue of $\boldsymbol{T}$. We inquire as to which $y$ lead to the value $\lambda_{1}$ for this reciprocal radius of convergence. (The largest positive eigenvalue of $T$ is $\lambda_{1}[8]$.)

It is known that $R_{\lambda} y$ is singular at $\lambda=\lambda_{1}$ if, and only if, $P_{1} y \neq 0$, where $P_{1}$ is the projection onto $\mathcal{N}\left[\left(\lambda_{1}-T\right)^{v}\right]$ along $\mathcal{R}\left[\left(\lambda_{1}-T\right)^{v}\right], \mathcal{N}=$ null-space, $\mathcal{R}=$ range, $\nu=$ index of $T$ at $\lambda_{1}=$ smallest integer $k$ such that $\mathcal{N}\left[\left(\lambda_{1}-T\right)^{k}\right]=$ $\mathcal{N}\left[\left(\lambda_{1}-T\right)^{k+1}\right]$. But $P_{1} z$, for arbitrary $z \in B$, has the form [10]

$$
P_{1} z=\sum_{i=1}^{k} \sum_{j=1}^{\mu_{i}}\left(z,\left(\lambda_{1}-T^{*}\right)^{j-1} y_{i}^{*}\right)\left(\lambda_{1}-T\right)^{\mu_{i}-j} y_{i}
$$


where $\quad 1 \leqq \mu_{1} \leqq \cdots \leqq \mu_{k}=v, \quad\left(\lambda_{1}-T^{*}\right)^{\mu_{i}} y_{i}^{*}=\left(\lambda_{1}-T\right)^{\mu_{i}} y_{i}=0, \quad$ and the $\left(\lambda_{1}-T\right)^{\mu_{i}-j} y_{i},\left(\lambda_{1}-T^{*}\right)^{j} y_{i}^{*}$ form, respectively, a basis for $\mathcal{N}\left[\left(\lambda_{1}-T\right)^{v}\right]$ and $\mathcal{N}\left[\left(\lambda_{1}-T^{*}\right)^{v}\right]$.

Now we recall the theorem of Karlin [8] to the effect that $\left(T-\lambda_{1}\right)^{v-1} P_{1}$ is a positive operator. (A detailed proof of this result is given in [6].) On applying $\left(\boldsymbol{T}-\lambda_{1}\right)^{v-1}$ to (3), and using the lemma proved above, we conclude that, for $z \geqq 0,\left(T-\lambda_{1}\right)^{v-1} P_{1} z=0$ is equivalent to

$$
\left(z,\left|\left(\lambda_{1}-T^{*}\right)^{v-1} y_{i}^{*}\right|\right)=0 \text { for } \mu_{i}=v .
$$

Let $K_{1}$ be the set of $z$ in $K$ which also satisfy (4). Then $K_{1}$ is easily seen to be a cone. If $B_{1}=K_{1}-K_{1}$, then $B_{1}$ is a Banach space and its norm is semimonotonic relative to the order induced by $K_{1}$. Furthermore, it is fairly easy to show that $B_{1}$ is invariant under $T$, that the index at $\lambda_{1}$ of $T$ restricted to $B_{1}$ is $v_{1} \leqq v-1$, and that, for $z$ in $K,\left(T-\lambda_{1}\right)^{p} P_{1} z \neq 0$ for some $p$ such that $\nu_{1} \leqq p \leqq \nu-1$ is equivalent to $z \notin K_{1}$. We now apply the argument of the preceding paragraph to $T \mid B_{1}$. After at most $v$ such steps, we reach the conclusion that, for $z \geqq 0, P_{1} z=0$ is equivalent to

$$
\left(z,\left|\left(\lambda_{1}-T^{*}\right)^{p} y_{i}\right|\right)=0 \text { for } 1 \leqq p \leqq \mu_{i}, 1 \leqq i \leqq k .
$$

Denote by $K_{2}$ the cone consisting of those $z$ in $K$ such that (5) holds, and let $B_{2}=K_{2}-K_{2}$. Then $B_{2}$ is $T$-invariant. If $T \mid B_{2}$ is quasi-nilpotent, then the results already proved establish the theorem. Otherwise let $\tilde{\lambda}_{2}(>0)$ be the largest eigenvalue of $T \mid B_{2}$. Since $T \mid B_{2}$ has a $\left(K_{2}-\right)$ positive eigenvector, it must be the case that $\tilde{\lambda}_{2} \leqq \lambda_{2}$. But the positive eigenvector of $T$ associated with $\lambda_{2}$ is in $K_{2}$, hence $\lambda_{2} \leqq \tilde{\lambda}_{2}$. Thus $\tilde{\lambda}_{2}=\lambda_{2}$. Applying the process in the two preceding paragraphs to $T \mid B_{2}$, we conclude that, for $z \in K_{1}, P_{2} z=0$ is equivalent to the obvious condition corresponding to (5). The general proof of the theorem is accomplished by repetition of this procedure.

ACKNOWLEDGMENT. This work is an extension of a portion of a Ph.D. dissertation submitted to the University of New Mexico. The author gratefully acknowledges the benefit of many helpful discussions with his advisor, Professor G. Milton Wing.

\section{REFERENCES}

1. M. M. Day, Normed linear spaces, Ergebnisse der Mathematik und ihrer Grenzgebiete, Heft 21, Springer-Verlag, Berlin, 1958. MR 20 \#1187.

2. M. A. Krasnosel'skil̆, Positive solutions of operator equations, Fizmatgiz, Moscow, 1962; English transl., Noordhoff, Groningen, 1964. MR 26 \#2862; MR 31 \#6107.

3. P. R. Halmos, Measure theory, Van Nostrand, Princeton, N.J., 1950. MR 11, 504.

4. A. E. Taylor, Introduction to functional analysis, Wiley, New York, 1958. MR 20 \#5411. 
5. M. G. Kreĭn and M. A. Rutman, Linear operators leaving invariant a cone in a Banach space, Uspehi Mat. Nauk 3 (1948), no. 1 (23), 3-95; English transl., Amer. Math. Soc. Transl. (1) 10 (1962), 199-325. MR 10, 256; MR 12, 341.

6. P. Nelson, Jr., An investigation of criticality for energy-dependent transport in slab geometry, Ph.D. Dissertation, University of New Mexico, Albuquerque, New Mexico, 1969.

7. - Subcriticality for transport of multiplying particles in a slab, J. Math. Anal. Appl. 35 (1971), 90-104.

8. S. Karlin, Positive operators, J. Math. Mech. 8 (1959), 907-937. MR 22 \#4965.

9. H. H. Schaefer, Topological vector spaces, Macmillan, New York, 1966. MR 33 \#1689.

10. A. C. Zaanen, Linear analysis, North-Holland, Amsterdam, 1964.

Mathematics Division, Oak Ridge National laboratory, Oak Ridge, TenNESSEE 37830 